\title{
A Break-Even Analysis for Dementia Care Collaboration: Partners in Dementia Care
}

\author{
Robert O. Morgan, $P h D^{7}$, David M. Bass, $P h D^{2}$, Katherine S. Judge, PhD ${ }^{3}$, C. F. Liu, PhD 4,5 , \\ Nancy Wilson, MSW ${ }^{6,7}$, A. Lynn Snow, PhD ${ }^{8,9}$, Paul Pirraglia, MD, MPH ${ }^{10,11}$, \\ Maurilio Garcia-Maldonado, MD 7,12 , Paul Raia, $P h D^{13}$, N. N. Fouladi, PhD, MPH', and Mark E. Kunik, \\ $\mathrm{MD}, \mathrm{MPH}^{6,7,74}$
}

\begin{abstract}
The University of Texas School of Public Health, Houston, TX, USA; ${ }^{2}$ Benjamin Rose Institute on Aging, Cleveland, OH, USA; ${ }^{3} \mathrm{Cleveland} \mathrm{State}$ University, Cleveland, OH, USA; ${ }^{V}$ VA Puget Sound Health Care System, Seattle, WA, USA; ${ }^{5}$ University of Washington School of Public Health, Seattle, WA, USA; ${ }^{6}$ Houston VA HSR\&D Center for Innovations in Quality, Effectiveness and Safety, Houston, TX, USA; ${ }^{7}$ Baylor College of Medicine, Houston, TX, USA; ${ }^{3}$ Tuscaloosa Veterans Affairs Medical Center, Tuscaloosa, AL, USA; ${ }^{9}$ University of Alabama, Tuscaloosa, AL, USA; ${ }^{10}$ Providence Veterans Affairs Medical Center, Providence, RI, USA; "'Brown University Medical School, Providence, RI, USA; ${ }^{2}$ Beaumont Veterans Affairs CommunityBased Outpatient Clinic, Beaumont, TX, USA; ${ }^{13}$ Alzheimer's Association, Massachusetts/New Hampshire Chapter, Watertown, MA, USA; ${ }^{14}$ VA South Central Mental Illness Education, Research and Clinical Center, Houston, TX, USA.
\end{abstract}

BACKGROUND: Dementia is a costly disease. People with dementia, their families, and their friends are affected on personal, emotional, and financial levels. Prior work has shown that the "Partners in Dementia Care" (PDC) intervention addresses unmet needs and improves psychosocial outcomes and satisfaction with care.

OBJECTIVE: We examined whether PDC reduced direct Veterans Health Administration (VHA) health care costs compared with usual care.

DESIGN: This study was a cost analysis of the PDC intervention in a 30-month trial involving five VHA medical centers.

PARTICIPANTS: Study subjects were veterans $(N=434)$ 50 years of age and older with dementia and their caregivers at two intervention $(N=269)$ and three comparison sites $(N=165)$.

INTERVENTIONS: PDC is a telephone-based care coordination and support service for veterans with dementia and their caregivers, delivered through partnerships between VHA medical centers and local Alzheimer's Association chapters.

MAIN MEASURES: We tested for differences in total VHA health care costs, including hospital, emergency department, nursing home, outpatient, and pharmacy costs, as well as program costs for intervention participants. Covariates included caregiver reports of veterans' cognitive impairment, behavior problems, and personal care dependencies. We used linear mixed model regression to model change in log total cost post-baseline over a 1year follow-up period.

KEY RESULTS: Intervention participants showed higher VHA costs than usual-care participants both before and after the intervention but did not differ significantly regarding change in log costs from pre- to post-baseline periods. Pre-baseline log cost $(p \leq 0.001)$, baseline cognitive impairment $(p \leq 0.05)$, number of personal care

Received August 8, 2014

Revised January 10, 2015

Accepted January 20, 2015

Published online February 10, 2015 dependencies $(p \leq 0.01)$, and VA service priority $(p \leq 0.01)$ all predicted change in log total cost.

CONCLUSIONS: These analyses show that PDC meets veterans' needs without significantly increasing VHA health care costs. PDC addresses the priority area of care coordination in the National Plan to Address Alzheimer's Disease, offering a low-cost, structured, protocol-driven, evidence-based method for effectively delivering care coordination.

KEY WORDS: costs and cost analysis; dementia; veterans.

J Gen Intern Med 30(6):804-9

DOI: $10.1007 / \mathrm{s} 11606-015-3205-\mathrm{x}$

(C) Society of General Internal Medicine 2015

\section{INTRODUCTION}

Dementia is a costly disease-people with dementia, their families, and their friends are affected on personal, emotional, and financial levels. In 2010, 36 million persons worldwide had dementia, and this is projected to increase to 66 million by 2030. ${ }^{1}$ Most would agree that dementia interventions should primarily focus on quality of life. Yet, with the annual cost of caring for someone with dementia in North America estimated at $\$ 56,000$ for $2010,2,3$ costs cannot be ignored.

Partners in Dementia Care (PDC) is a telephone-based care coordination and support service intervention for veterans with dementia and their family caregivers, delivered through partnerships between Veterans Health Administration (VHA) medical centers and local Alzheimer's Association (AA) chapters. It was designed to be a feasible and practical intervention integrating health, community, and support services to address the unmet care needs of patients and their caregivers across all dementia stages. The Cleveland Alzheimer's Managed Care Demonstration and the Chronic Care Networks for Alzheimer's Disease laid the groundwork for the current PDC intervention. ${ }^{4-6}$ PDC has been shown to lessen dementia- 
related needs and improve psychosocial outcomes for patients and caregivers. $^{4-9}$

Notwithstanding the demonstrated effectiveness of PDC in reducing distress for both veterans with dementia and their caregivers, providers such as the VHA cannot make policy decisions on the basis of effectiveness alone. The literature on cost savings of non-pharmacologic therapies, including those focusing on organization of care, is relatively small and inconclusive. $^{10,11}$ Past studies of interventions attempting to better coordinate and manage dementia care have shown mixed impacts on use of services. ${ }^{12}$ Fewer studies have focused on health care costs.

PDC was a 5-year research investigation (2006-2011) that tested the effectiveness of an innovative care coordination intervention for veterans with dementia and their family caregivers. Shared care coordination between a VHA and communitypartner agency was intended to enhance access to VHA and non-VHA services and to improve the integration of medical care and the network of community service organizations. The objective of these analyses was to examine the impact of PDC on direct care costs for veterans with dementia within the VHA system in order to determine whether the VHA "broke even" with respect to health care costs for participants in the PDC intervention. In the context of a clinically successful program such as PDC, we posited that findings that were either costneutral or cost-reducing could be interpreted as "cost successful" for a health care system such as the VHA. For a complete description of the PDC protocol, see Judge et al. ${ }^{13}$

\section{METHODS}

Sample Design. As described elsewhere, ${ }^{14}$ participants were recruited over 2.5 years from five different communities. Boston, MA, and Houston, TX, served as PDC sites; and Providence, RI, Beaumont, TX, and Oklahoma City, OK, served as control (usual-care) sites. All are located in one of two selected Veterans Integrated Service Networks (VISNs), which provide a unifying administrative structure for all VHA services within a given geographic region. Study sites were matched by VISN to ensure uniformity in this overarching administrative structure. VISN 16, which includes Houston, Oklahoma City, and Beaumont, was chosen because it was the location of the study's VHA principal investigator. The other selected VISN (VISN 1), which includes Boston and Providence, had an array of VHA and Alzheimer's Association services similar to VISN 16.

Recruitment for PDC included veterans with a new or preexisting dementia diagnosis (Table 1) and their caregivers. Veteran eligibility criteria included receiving primary care from the VHA, living outside an institutional setting, and being $50+$ years of age. Eligibility was not restricted based on dementia severity or symptoms. Caregiver eligibility criteria included being an unpaid family member or friend and providing the most assistance with veterans' personal care,
Table 1 ICD-9 Diagnosis Codes Used to Identify Veterans with Dementia

\begin{tabular}{|c|c|}
\hline $\begin{array}{l}\text { ICD-9 } \\
\text { diagnostic } \\
\text { code }\end{array}$ & Description \\
\hline 290.xx & Pre-senile and senile dementia, vascular dementia \\
\hline 291.2 & Alcohol-induced persistent dementia \\
\hline 292.82 & Drug-induced persistent dementia \\
\hline $294.1 \mathrm{x}$ & $\begin{array}{l}\text { Dementia in conditions classified elsewhere with/ } \\
\text { without behavioral disturbance }\end{array}$ \\
\hline $294.2 \mathrm{x}$ & $\begin{array}{l}\text { Dementia, unspecified, with/without behavioral } \\
\text { disturbance }\end{array}$ \\
\hline 294.8 & $\begin{array}{l}\text { Other persistent mental disorders due to conditions } \\
\text { classified elsewhere }\end{array}$ \\
\hline 331.0 & Alzheimer's disease \\
\hline
\end{tabular}

daily living tasks, and/or health-related decisions. Although PDC targeted recruitment of veteran-caregiver dyads, veterans with dementia could enroll without a caregiver. ${ }^{13}$ A total of 1,726 veterans were referred to the project and mailed IRBapproved study information and consent forms. Signed consent forms were received for 508 veterans and 486 caregivers.

Intervention. PDC was created to address major issues reported by older adults with chronic illnesses and their caregivers, including fragmentation and lack of coordination between medical care and community services as well as difficulties among families in accessing and monitoring the quality of medical and non-medical services. ${ }^{6,15}$ PDC assisted families by 1) providing disease-related education and information, 2) offering emotional support and coaching, 3) linking families to medical and non-medical services and resources, and 4) mobilizing and organizing the informal care network. The intervention was implemented by a half-time VHA dementia care coordinator (DCC) and a half-time AA care consultant (CC). DCCs and CCs were bachelor's or master's degree-prepared social workers or nurses. Each DCC/CC pair worked together. With part-time administrative support, they maintained a caseload of approximately 100 to 120 caregiving dyads. VHA DCCs primarily focused on veterans' medical and non-medical needs and assisted families with effectively using VHA resources; AA CCs primarily focused on the needs of informal caregivers, such as care-related strain and accessing non-VHA resources.

The intervention consisted of 1) assessment of care needs across medical and non-medical care issues that addressed 23 domains for veterans and 14 domains for caregivers; 2) development of care goals that matched the priorities of veterans and caregivers; 3) development of action steps, concrete behavioral tasks intended to help families move toward goal achievement; and 4) ongoing monitoring of action steps on a regular basis. Patients and caregivers in both the PDC and usual care arms received printed materials about dementia. ${ }^{16}$

VHA Data. VHA health service cost data were obtained from the national data extract files of the VHA's Decision Support 
System, or DSS. DSS costs reflect actual estimated costs to VHA facilities as opposed to reimbursements from a payer (e.g., Medicare). DSS costs are based on a relative value unit methodology for allocating facility costs extracted from payroll and general ledger accounts (http://www.herc. research.va.gov/data/dss.asp). As such, they reflect regional differences in costs. Cost data in this study included inpatient, nursing home, outpatient, and pharmacy costs. Our final total cost measure was the sum of component costs. Costs were extracted for 1 year prior to and 1 year after each participant's baseline interview.

Our measure of overall health burden, the Charlson/Deyo index, was calculated from pre-baseline utilization data. Each veteran's VHA priority for service score was also collected from VHA administrative data. A veteran's priority score, based on his/her service and/or service-connected disability, determines the extent of his/her health care coverage within the VHA medical system. Priority scores range from $1-8 b$, with 1 being the highest service priority. For this study, veterans were grouped into three sets of priority levels (i.e., 1, 2-6, and 7a-8b), broadly differentiating copayment levels and out-of-pocket maximums. ${ }^{17}$

The intervention cost to deliver PDC (e.g., coordinator salaries, benefits, equipment, supplies, training, software, licensing, supervision, and administrative overhead) was estimated at $\$ 65$ to $\$ 80$ per month per dyad ( $\$ 780$ to $\$ 960$ per year), depending on the caseload for the care coordination team. DCCs, CCs, and dyads averaged 24.6 contacts during the 12-month study period ( $\mathrm{SD}=15.4)$.

Patient Symptom and Background Data. Caregivers completed a structured telephone interview at study baseline, conducted by trained research staff. Three multi-item scales represented caregiver reports of veterans' symptoms at the time of study enrollment: 1) cognitive impairment, 2) behavior problems, and 3) personal care dependency. ${ }^{18,19}$

In addition, five background or context characteristics were collected to control for baseline differences. These included patient and caregiver age, whether the caregiver was a spouse versus other relative of the veteran, and whether the caregiver was from a northeast or southwest study site. We also used the number of days between the dementia diagnosis and date of baseline interview as a covariate in our analyses.

Analysis. All analyses were conducted using SAS 9.2 (SAS Institute, Inc., Cary, NC, USA). With the exceptions noted below, participants were included in the analyses regardless of the extent to which they participated in the intervention. We excluded participants who died during the follow-up period, since their cost data were aggregated over different periods compared with participants who completed the 1-year follow-up.

Differences in the baseline characteristics of intervention- and control-site participants were examined using Wilcoxon rankorder tests for the continuous measures, and contingency table analysis with a chi-square test statistic for categorical measures.
The distribution of our total cost measure was highly skewed. Multiple approaches have been described in the literature for analyzing skewed cost data. Manning and Mullahy provide an algorithm for choosing among some of the frequently used alternatives based on a comparison of residuals. ${ }^{20}$ Based on their algorithm, we modeled change in total cost using a generalized linear mixed model regression with change in log cost as a normally distributed dependent variable. A small percentage of our participants had zero-dollar costs post-baseline ( $<2 \%$ of participants). These participants were distributed between intervention and control sites and were dropped from analyses. Our model included the corresponding pre-intervention measure of log-transformed total cost as a covariate. Consequently, our model was an analysis of intervention- versus control-group differences in adjusted pre- to post-intervention change.

Re-transforming estimated log costs can be problematic due to potential unequal variability in model residuals (heteroskedasticity) across the range of the predictors. ${ }^{21,22}$ We used a residual-based adjustment method to obtain unbiased estimates of adjusted costs accounting for heteroskedasticity in the log cost model residuals. ${ }^{21}$

Finally, individuals were clustered within study sites. For purposes of our models, the study site was treated as a random variable, and sites were nested within their study arm (intervention or control). We conducted a separate mixed-model logistic regression analysis of risk of mortality between intervention and control participants to determine whether there were different mortality rates between study arms. We used SAS PROC MIXED and PROC GLIMMIX for our analyses.

\section{RESULTS}

Sample Characteristics. During the 1-year follow-up period, 36 of $316(11.4 \%)$ intervention participants and 18 of 192 $(9.4 \%)$ control participants died. This difference was nonsignificant, both unadjusted and adjusted for baseline characteristics, indicating no difference in attrition due to death. The 54 study participants who died during follow-up were excluded from our analysis. In addition, we excluded 12 veterans for whom we did not have VHA service priority scores and nine veterans who had no VHA health care costs during the postintervention period (one veteran was missing both). Our final sample size was 434 .

There were no differences in ages of participants between the intervention and control ( $79.1 \pm 8.3$ versus $80.3 \pm 6.3$ years), although the spouses of the intervention participants were younger than those of the control participants $(68.2 \pm 12.3$ versus $71.3 \pm$ $10.6 ; p \leq 0.01)$. The intervention participants were less likely to have spouse caregivers (64.3\% versus $79.3 \% ; p \leq 0.001)$ and more likely to have higher average pre-baseline Charlson-Deyo index scores (2.6 \pm 2.4 versus $1.8 \pm 1.7 ; p \leq 0.01)$. 
Table 2 Pre- and Post-Intervention Change in Total Health Care Costs

\begin{tabular}{lll}
\hline \hline & $\begin{array}{l}\text { Unadjusted change } \\
\text { (pre-intervention } \\
\text { through follow-up) }\end{array}$ & $\begin{array}{l}\text { Adjusted change } \\
\text { (pre-intervention } \\
\text { through follow-up) }\end{array}$ \\
\hline Control $(n=165)$ & $\$ 957(\$ 10,837)^{\mathrm{a}}$ & $\$ 2,540(\$ 4,771)$ \\
Intervention $(n=269)$ & $\$ 3,235(\$ 24,242)$ & $\$ 3,546(\$ 11,616)$ \\
Difference in difference & $\$ 2,278(\$ 20,221)$ & $\$ 1,006(\$ 9,607)$ \\
\hline
\end{tabular}

${ }^{a}$ Costs are shown as mean (SD)

There were no differences between intervention and control participants at baseline in average number of days between diagnosis and the baseline interview, number of activities of daily living (ADLs) they needed assistance with, their behavior problems score, or their level of cognitive impairment.

Cost. The unadjusted and adjusted total costs pre- and postbaseline are shown in Table 2. As described above, our decision to log-transform the costs was made using the methods described by Manning and Mullahy. ${ }^{20}$ Adjusted costs were then estimated, based on our model results, using the residual-based retransformation methods described by Baser. ${ }^{21}$ Notably, there was no evidence of heteroskedasticity related to study arm.

Both the unadjusted and adjusted total costs were greater for intervention participants than for controls; however, the magnitude of difference in the post-baseline costs was much smaller, and statistically nonsignificant, for the adjusted total costs.

The results from our multivariable regression on postintervention log costs are shown in Table 3. Although PDC participants showed higher costs before and after intervention,

Table 3 Predictors of Change in Log-Total Costs ${ }^{\mathrm{a}}$

\begin{tabular}{lll}
\hline \hline $\mathbf{N = 4 3 4}$ & Estimate & $\begin{array}{l}\text { Standard } \\
\text { error }\end{array}$ \\
\hline Parameter & & 0.75 \\
& 4.01 & 0.23 \\
Intercept & -0.15 & \\
Control & Ref & -0.05 \\
Intervention & $-0.42 * * * \mathrm{~b}$ & 0.05 \\
Pre-baseline cost (log) & 0.03 & 0.02 \\
Charlson/Deyo & -0.00 & 0.01 \\
Patient age & -0.00 & 0.01 \\
Caregiver age & -0.06 & 0.14 \\
Spouse caregiver (No) & Ref & $\cdot$ \\
Spouse caregiver (Yes) & 0.00 & 0.00 \\
Days between diagnosis and intervention & 0.07 & 0.22 \\
PDC region (South) & Ref &. \\
PDC region (North) & $0.05^{* *}$ & 0.02 \\
ADLs & 0.02 & 0.02 \\
Behavioral problems & $-0.03^{*}$ & 0.01 \\
Cognitive impairment & 0.32 & 0.17 \\
Priority group (1) & $0.34^{* * *}$ & 0.10 \\
Priority group (2-6) & Ref & $\cdot$ \\
Priority group (7,8) & & \\
\hline
\end{tabular}

${ }^{a}$ We used generalized linear mixed model regression with change in log cost as the dependent variable. Individuals were nested within the PDC site, with site treated as a random variable and nested within the intervention arm

${ }^{b} p \leq 0.05\left(^{*}\right), p \leq 0.01\left(^{* *}\right), p \leq 0.001$ (***) $^{* *}$

${ }^{c} A D L s$ activities of daily living the change in transformed costs from pre- to post-baseline was not statistically significant. The strongest predictor of change in cost was the pre-baseline total cost.

The other significant predictors were the baseline level of cognitive impairment, with greater impairment negatively associated with change in cost; the number of functional impairments (ADLs), with greater number of impairments positively associated with change in cost; and VHA priority group, with priority grouping 2-6 showing greater cost increases than groups of veterans with lower priority (priority 7 and above).

The cost of PDC was estimated to range between $\$ 780$ and $\$ 960$ per dyad over a 12-month period. The difference in change in log-total cost between intervention and control conditions remained statistically nonsignificant when intervention costs were included in the total costs for the intervention participants.

\section{DISCUSSION}

Generally, reviews of dementia care research have found little available evidence on the cost effectiveness of care management or care organization interventions. ${ }^{11,23}$ This study examined the impact of the PDC intervention on VHA health care costs. In our study, the model-adjusted costs for veterans receiving the PDC intervention were not significantly higher than those for the veterans in the control arm, while, as we have documented elsewhere, veterans and their caregivers receiving PDC showed improvements in care outcomes. $^{7-9}$

Similar to the results in our study, those reported by Duru et al., ${ }^{24}$ who examined the implementation of a social workeroriented intervention focused on problem and action plan prioritization, identified improvements in care quality but no differences in health care costs. Wray et al. ${ }^{25}$ noted an average decrease in total costs of \$2,768/patient over 6 months, with a statistically significant reduction in costs for veterans in their intervention group (Telehealth Education Program [TEP]) over the intervention period and immediately following the intervention. However, the total cost decrease variable was not maintained over the subsequent 6 months, possibly because the TEP was not continued beyond the completion of the 10week program. The authors speculate that improvements related to the TEP program might have been maintained if ongoing support had been provided for caregivers.

Studies of health care costs in case management and collaborative care interventions for depression are more numerous. Encouragingly, costs for such interventions may be frontloaded. A review by Gilbody et al. ${ }^{26}$ reported general findings of improved care outcomes accompanied by increased health care costs over the short term (6-12 months); however, these increased short-term costs dissipated over a 28 -month followup. ${ }^{27}$ Their findings are heartening in light of the similarity to what we have seen with PDC; however, they suggest that it may be important to track costs over longer periods than those used in our study. Long and colleagues ${ }^{28}$ projected that the 
savings from a successful dementia caregiver intervention could be substantial over the long term (15-year period), but that it might take several years to begin accruing.

Limitations. A major limitation of this study was the lack of randomization within sites. There were substantial site-related differences in cost among PDC and control participants prior to implementing the intervention, which suggests that some differences in use are driven by differences in patient comorbidity levels or in the service structure across the individual sites. We attempted to account for these site effects by using a within-patient difference-in-difference approach, controlling for comorbidities, and by incorporating site as a random effect in our models. Further, we incorporated a measure of cost prior to PDC implementation as a covariate to help adjust for historical use by individual participants.

A second limitation was our lack of measures of non-VHA health care use. Veterans are known to use both VHA and nonVHA health care, ${ }^{29}$ and it is possible that veterans with less service priority may be more likely to seek care outside the VHA system. We incorporated an indicator of veteran priority levels to help control for the impact of service priority on change in VHA cost. Wray et al. ${ }^{25}$ concluded that Medicare use in their study by veterans with dementia was minimal compared to VHA use among the same group of veterans. However, without measures of non-VHA health care use, we cannot determine whether PDC affected VHA care and non-VHA care access differentially. Similarly, the VHA DSS indicators of health care costs reflect resource utilization useful for management decision-making, and while ideal for our purposes, they are derived in a different manner from the reimbursement-based indicators frequently used in analyses of non-VHA data (e.g., Medicare). Consequently, our cost estimates may not translate directly to non-VHA health care systems.

Third, the difference-in-difference effect we observed was very small, $<0.1 \mathrm{SD}$ in the log scale, and approximately $0.1 \mathrm{SD}$ for the re-transformed adjusted change in costs (Table 2). Our study was powered to detect small to moderate differences in change in cost, on the order of those observed by Wray et al. ${ }^{25}$ Further work with substantially larger samples could help to determine whether the small cost differences we observed exist.

Finally, while the PDC study overall has documented improvements in clinical outcomes for veterans' caregivers, ${ }^{7}$ we did not assess health care use by caregivers. Consequently, it is not clear whether PDC affected caregivers' health care use, or, if so, in which direction.

\section{CONCLUSIONS}

Our objective in these analyses was to determine whether the VHA "broke even" with respect to health care costs for participants in the PDC intervention. Our conclusion is that PDC demonstrated significant improvements in psychosocial outcomes, without substantially increasing costs, over a 1year follow-up period compared with usual care. Patients with dementia and their family caregivers have high unmet medical and psychosocial needs, which exacerbate the difficulties of coordinating medical care and needed community, support, and information services. PDC maintained near cost neutrality while addressing many of these unmet needs and improving satisfaction with care and psychosocial outcomes for patients and their caregivers. ${ }^{14}$ This combination of findings suggests that PDC is a promising model for care coordination, although further work is needed to determine whether cost neutrality can be maintained over the longer term.

Further, PDC addresses the priority area of care coordination in the recently enacted National Plan to Address Alzheimer's Disease and closely matches the recently proposed amendment to the Older Americans Act calling for care coordination to link health care services and community service networks for older adults and their caregivers. PDC offers these national initiatives a structured, protocol-driven, evidence-based method for effectively delivering care coordination for patients with dementia, and appears to do so while controlling health care costs.

ACKNOWLEDGMENTS: This work was supported by a grant from the Department of Veterans Affairs, Health Services Research and Development (HR 04-238-3), and grants from the Alzheimer's Association (IIRG-08-89058) and the Robert Wood Johnson Foundation (\#57816). This work was supported in part by the Houston VA HSR\&D Center for Innovations in Quality, Effectiveness \& Safety [CIN 13-413]. The attitudes expressed herein are those of the authors and do not necessarily reflect those of the Department of Veterans Affairs/ U.S. government or Baylor College of Medicine. The funders had no role in the study design, data collection and analysis, decision to publish, or preparation of the manuscript.

Conflicts of Interest: The authors report no conflicts of interest.

Corresponding Author: Robert O. Morgan, $\mathrm{PhD}$; The University of Texas School of Public Health, Houston, TX, USA (e-mail: Robert.O.Morgan@uth.tmc.edu).

\section{REFERENCES}

1. World Alzheimer Report 2009. Alzheimer Disease International, 2009. Available at www.alz.org/national/documents/report_full_2009worldalzheimer. Accessed July 28, 2014.

2. Hurd MD, Martorell P, Delavande A, Mullen KJ, Langa KM. Monetary costs of dementia in the United States. N Engl J Med. 2013;368(14):1326-1334.

3. World Alzheimer Report 2010. The Global Economic Impact of Dementia. Alzheimer Disease International, 2010. Available at http://www.alz.org/ documents/national/world-alzheimer-report-2010.pdf. Accessed July 28, 2014.

4. Bass DM, Clark PA, Looman WJ, McCarthy CA, Eckert S. The Cleveland Alzheimer's Managed Care Demonstration: outcomes after 12 months of implementation. Gerontologist. 2003;43(1):73-85.

5. Bass DM, Maslow K. Model 4: National Chronic Care Consortium/ Alzheimer's Association Managed Care and Dementia project. Paper presented at the Symposium preconference at the 56th annual meeting of the Gerontological Society of America, San Diego, CA, Nov. 21-25, 2003.

6. Maslow K, Selstad J. Chronic care networks for Alzheimer's disease: Approaches for involving and supporting family caregivers in an innovative model of dementia care. Alzheimer's Care Today. 2001;2(1):33-46.

7. Bass DM, Judge KS, Snow AL, et al. Caregiver outcomes of partners in dementia care: effect of a care coordination program for veterans with 
dementia and their family members and friends. J Am Geriatr Soc. 2013;61(8):1377-1386

8. Bass DM, Judge KS, Snow AL, et al. A controlled trial of Partners in Dementia Care: veteran outcomes after six and twelve months. Alzheimers Res Ther. 2014;6(1):9.

9. AHRQ Innovations Exchange. Partnership Supports Patients With Dementia and Their Caregivers Through Care Coordinator Team, Leading to Improved Psychosocial Outcomes. Agency for Healthcare Research and Quality, 2012, from http://www.innovations.ahrq.gov/content.aspx?id=3345.

10. Goy E, Kansagara D, Freeman M. VA Evidence-based Synthesis Program Reports. A Systematic Evidence Review of Interventions for Nonprofessional Caregivers of Individuals with Dementia. Washington, DC: Department of Veterans Affairs; 2010.

11. Knapp M, Iemmi V, Romeo R. Dementia care costs and outcomes: a systematic review. Int J Geriatr Psychiatry. 2013;28(6):551-561.

12. Pimouguet C, Lavaud T, Dartigues JF, Helmer C. Dementia case management effectiveness on health care costs and resource utilization: a systematic review of randomized controlled trials. J Nutr Health Aging. 2010;14(8):669-676.

13. Judge KS, Bass DM, Snow AL, et al. Partners in dementia care: a care coordination intervention for individuals with dementia and their family caregivers. Gerontologist. 2011;51(2):261-272.

14. Steiger-Gallagher K, Bass DM, Judge KS, et al. Satisfaction with dementia care. Fed Pract. 2012;29(4):33-40.

15. Eloniemi-Sulkava U, Saarenheimo M, Laakkonen ML, et al. Family care as collaboration: effectiveness of a multicomponent support program for elderly couples with dementia. Randomized controlled intervention study. J Am Geriatr Soc. 2009;57(12):2200-2208.

16. Understanding Memory Loss. What to Do When You Have Trouble Remembering. National Institute of Aging, May 2013. Available at http:// www.nia.nih.gov/sites/default/files/understanding_memory_loss.pdf. Accesses August 5, 2014

17. Stroupe KT, Smith BM, Lee TA, et al. Effect of increased copayments on pharmacy use in the Department of Veterans Affairs. Med Care. 2007:45(11):1090-1097.
18. Bass DM, McClendon MJ, Deimling GT, Mukherjee S. The influence of a diagnosed mental impairment on family caregiver strain. J Gerontol. 1994;49(3):S146-S155.

19. Lawton MP, Brody EM. Assessment of older people: self-maintaining and instrumental activities of daily living. Gerontologist. 1969;9(3):179-186.

20. Manning WG, Mullahy J. Estimating log models: to transform or not to transform? J Health Econ. 2001;20(4):461-494.

21. Baser O. Modeling transformed healthcare cost with unknown heteroskedasticity. App Econ Res Bull. 2007;01:106.

22. Duan N. Smearing estimate: A nonparametric retransformation method. J Am Stat Assoc. 1983;78(383):605-610.

23. Koch T, Iliffe S, Manthorpe $\mathbf{J}$, et al. The potential of case management for people with dementia: a commentary. Int $\mathrm{J}$ Geriatr Psychiatry. 2012;27(12):1305-1314.

24. Duru OK, Ettner SL, Vassar SD, Chodosh J, Vickrey BG. Cost evaluation of a coordinated care management intervention for dementia. Am J Manag Care. 2009; 15(8):521-528.

25. Wray LO, Shulan MD, Toseland RW, Freeman KE, Vásquez BE, Gao J. The effect of telephone support groups on costs of care for veterans with dementia. Gerontologist. 2010;50(5):623-631.

26. Gilbody S, Bower P, Whitty P. Costs and consequences of enhanced primary care for depression: systematic review of randomised economic evaluations. Br J Psychiatry. 2006;189:297308.

27. Katon W, Russo J, Von Korff M, et al. Long-term effects of a collaborative care intervention in persistently depressed primary care patients. J Gen Intern Med. 2002;17(10):741-748.

28. Long KH, Moriarty JP, Mittelman MS, Foldes SS. Estimating the potential cost savings from the New York University Caregiver Intervention in Minnesota. Health Aff (Millwood). 2014;33(4):596604.

29. Byrne MM, Kuebeler M, Pietz K, Petersen LA. Effect of using information from only one system for dually eligible health care users. Med Care. 2006;44(8):768-773. 